\title{
Caracterización del comportamiento ocupacional de personas desplazadas por la violencia ${ }^{1}$
}

\author{
Marisol Gómez Oliveró \\ Sara Elena Torres Mogollón ${ }^{3}$ \\ María Teresa Rodriguez García ${ }^{4}$
}

\section{RESUMEN}

La investigación realizada con el fin de caracterizar el Comportamiento Ocupacional de personas desplazadas por la violencia, corresponde a un estudio social de tipo cualitativo, que analiza las características y cambios ocupacionales de una población compuesta por cuarenta jefes o cabezas de hogar, entre los que se incluyen hombres y mujeres, ubicados en el barrio Divino Niño en la localidad 19 de Ciudad Bolivar, en Santa Fe de Bogotá, que han sido desplazados por la violencia de zonas afectadas por el recrudecimiento de la guerra. El trabajo se dirige a los jefes de hogar, puesto que son éstos quienes poseen y mantienen el rol de proveedor, y por la naturaleza de sus ejecuciones ocupacionales, son los que evidencian con mayor claridad los cambios ocupacionales ocurridos a partir de la experiencia del desplazamiento; sin embargo, sus familias fueron involucradas dentro de determinadas acciones desarrolladas a lo largo de la investigación, con el fin de promover la integración familiar y social y el establecimiento de canales de comunicación entre comunidad y profesionales, en un proceso mutuo de retroalimentación.

1 Trabajo de Grado para optar al título de Terapeuta Ocupacional. Mención Meritoria. Universidad Nacional de Colombia. Diciembre de 1998. Primer Lugar Concurso Mejores Trabajos de Grado IX Versión 1998-1999 Área Ciencia de la Salud, Universidad Nacional.

2 Terapeuta Ocupacional, Universidad Nacional de Colombia.

3 Terapeuta Ocupacional, Universidad Nacional de Colombia.

- Profesora Asociada, Departamento de Terapias, Universidad Nacional de Colombia. Directora Trabajo de Grado. 
Se enumeran los aspectos que enmarcan el fenómeno del desplazamiento, las acciones nacionales $e$ internacionales en materia, y la intervención del Terapeuta Ocupacional en esta temática. A la luz del Modelo Teórico de la Ocupación Humana, planteado por Gary Kielhofner, se realizó un análisis detallado de sus componentes, observando la influencia de dicho fenómeno en el bienestar ocupacional de las personas afectadas, en términos de la adquisición de nuevos roles y transformación de patrones de vida, reflejados en su cotidianidad. Se encontró que los Procesos Ocupacionales de Trabajo y Tiempo Libre presentan marcados cambios a raíz de la experiencia del desplazamiento, y que las consecuencias del evento generan pobres expectativas de vida frente al futuro. Se incluyen recomendaciones con el propósito de profundizar en el estudio de temáticas álgidas acordes con la realidad social del país, y en las cuales el Terapeuta Ocupacional debe aumentar su participación.

\section{SUMMARY}

The research was made to characterize the occupational behavior of people who migrate for violence. It is a cualitative social studio that examines the occupational characteristics and occupational changes of a group of 40 home heads, men and women, who live in the "Divino Niño" neighborhood, in Ciudad Bolivar, in Santafé de Bogotá. They migrate for the violence from their towns, because the war makes their lifes worse.

The research is oriented to home heads, because they have and keep the role as provider, and for their occupational executions, they show better the occupational changes after the experience of migration for violence; however, their families participated in several activities, for promoting the familiar and social integration and establishing communication channels between the community and the professionals, in a feedback process.

The aspects of the migration for violence, the national and international politics for this topic, and the participation of the Occupational Therapist, are explained.

According to the Theoretical Model of Human Occupation (Gary Kielhofner), a detailed analysis about its components, the influence of the migration for violence in the occupationl well-being of the victims, and the acquisition of new roles were carried out; also, the transformation of lifestyles in their daily lifes were studied. It was observed that the Occupational Processes of Working and Free Time have important changes for the experience of the migration for violence, and its 
consequences originate less life prospects for the future.

Some recommendations are suggested for increasing the studio of social topics related with the national reality where the Occupational Therapist must participate actively.

\section{INTRODUCCIÓN}

El desplazamiento forzado por la violencia constituye un problema de actualidad nacional, de gran magnitud social, violatorio de los Derechos Humanos, frecuentemente evadido en su atención y con solución exigible al estado, el cual requiere estudios para su caracterización y recomendaciones para su superación. Según el último estudio de la Consejería Presidencial para los Desplazados, durante 1997 y julio de 1998, se desplazaron en el país 912.114 personas procedentes de 177.169 hogares, originarios de zonas rurales, principalmente de los departamentos de Antioquia, Santander, Meta y Córdoba. (1)

La dinámica del desplazamiento forzado ha sido ampliamente estudiada dentro de las áreas sociales, encontrándose importantes hallazgos; sin embargo, desde la perspectiva de la salud, no existen estudios en esta materia, lo que constituyó la principal motivación para abordar dicha temática.

Terapia Ocupacional al ser una profesión que tiene como objeto de estudio el Desempeño Ocupacional del individuo, se interesa en comprender e intervenir en la solución de problemas que lo afectan de manera integral, yendo más allá del modelo clínico para reconocer el efecto de las dinámicas sociales cambiantes sobre el comportamiento del individuo y su calidad de vida, dentro de la esfera familiar y social.

\section{PROCESO METODOLÓGICO}

Primera fase: Revisión bibliográfica de la literatura existente en materia de derechos humanos, violencia, desplazamiento forzado y acciones legales de carácter nacional e internacional al respecto. Se contemplan aspectos como las características, áreas de concentración y lugares de migración, motivos, responsables y consecuencias del desplazamiento. 
Segunda fase: Establecimiento del contacto interpersonal con la población objeto, reconocimiento del área de trabajo y sus condiciones en cuanto a salubridad, marginalidad, servicios públicos y vías de acceso, entre otros. Se caracteriza la población objeto en cuanto a lugar de procedencia, distribución por sexo, intervalo de edades, nivel de escolaridad, permanencia en Santa Fe de Bogotá, permanencia en el barrio Divino Niño, tenencia de vivienda, responsables y motivos del desplazamiento, y deseo de retorno. Se presentan a la comunidad los objetivos del trabajo a desarrollar, y se indaga acerca de las expectativas frente al mismo.

Tercera fase: Abordaje de la temática con la población, mediante el establecimiento de diálogos informales, es decir, conversatorios con directrices establecidas de antemano, sin presentar un cuestionario a la población objeto, debido a la dificultad de obtener información de los sucesos violentos en que el individuo se vio envuelto. Mediante los diálogos se busca conocer la cotidianidad en los Procesos Ocupacionales de Trabajo y Tiempo Libre, intereses ocupacionales y expectativas de vida de las personas. Igualmente sondear acerca de los factores desencadenantes del fenómeno y responsables del mismo.

Cuarta fase: Realización de cinco talleres con la comunidad, que fueron realizados en uno de los Hogares Comunitarios del barrio Divino Niño, utilizando la técnica de reunión en mesa redonda, con presentación de objetivos, actividades a desarrollar e identificación de las expectativas de los asistentes frente al mismo; luego se realiza la exposición del tema por parte de las Terapeutas, con simultánea retroalimentación de los asistentes a lo largo de la presentación; distribución del material pertinente a cada taller, conocimiento de necesidades de la población con el fin de planear temáticas para ser abordadas en próximos encuentros; finalmente, suministro de refrigerio a los asistentes.

Se abordaron temáticas tales como oportunidades laborales, manejo y resolución de conflictos, formas de organización comunitaria y manejo productivo del tiempo libre.

La técnica de talleres fue utilizada debido a que facilita la obtención de información, genera mayor participación a nivel comunitario, promueve niveles de socialización y permite aportar por parte de los profesionales, sugerencias para solucionar las situaciones presentes que afectan a la comunidad, y de esta manera contribuir al mejoramiento de la calidad de vida individual y colectiva. 
Quinta fase: Interpretación de la información obtenida a la luz del Modelo Teórico de la Ocupación Humana, presentando así una completa descripción de los cambios ocupacionales y el desarrollo de los roles mantenidos y/o adoptados en la actual situación. La interpretación cualitativa de los datos constituye la base de la caracterización del Comportamiento Ocupacional de la población objeto.

Sexta fase: Encuentro final con la comunidad con el objeto de dar a conocer los resultados del trabajo conjunto realizado a lo largo de la investigación. Este espacio permitió ratificar la importancia de la intervención de Terapia Ocupacional, dar continuidad a las actividades desarrolladas y mantener el contacto interpersonal con los miembros de la comunidad.

\section{DESCRIPCIÓN DE LA POBLACIÓN OBJETO}

Como producto de la investigación, se presenta una primera caracterización de la población objeto en cuanto a sus características generales tales como procedencia, sexo, edad, escolaridad, permanencia en Santa Fe de Bogotá y en el barrio Divino Niño, responsables y motivos del desplazamiento, y deseo de retorno. Tal caracterización es presentada en las siguientes gráficas:

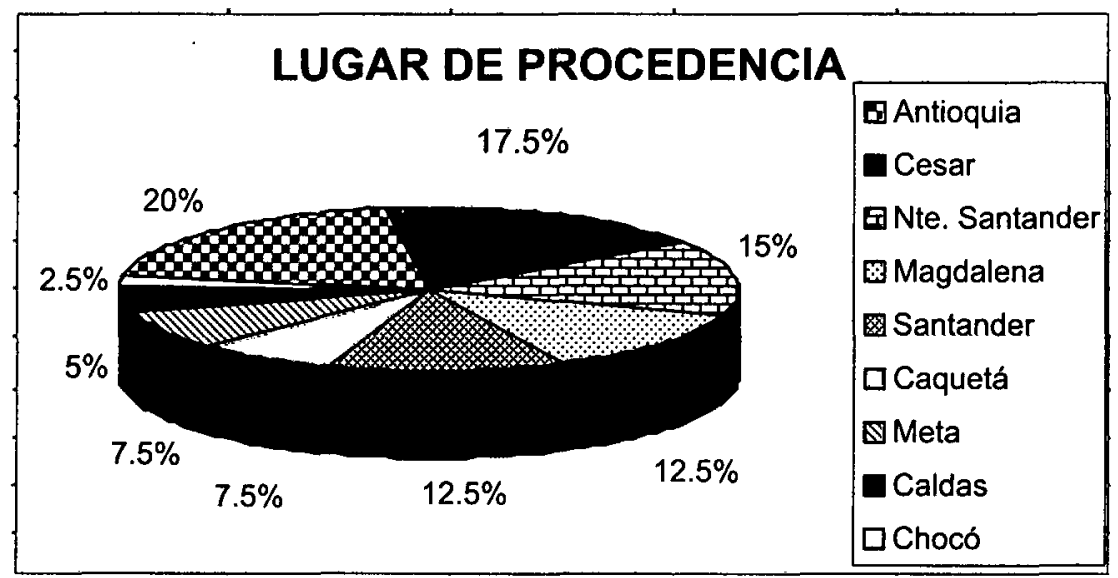



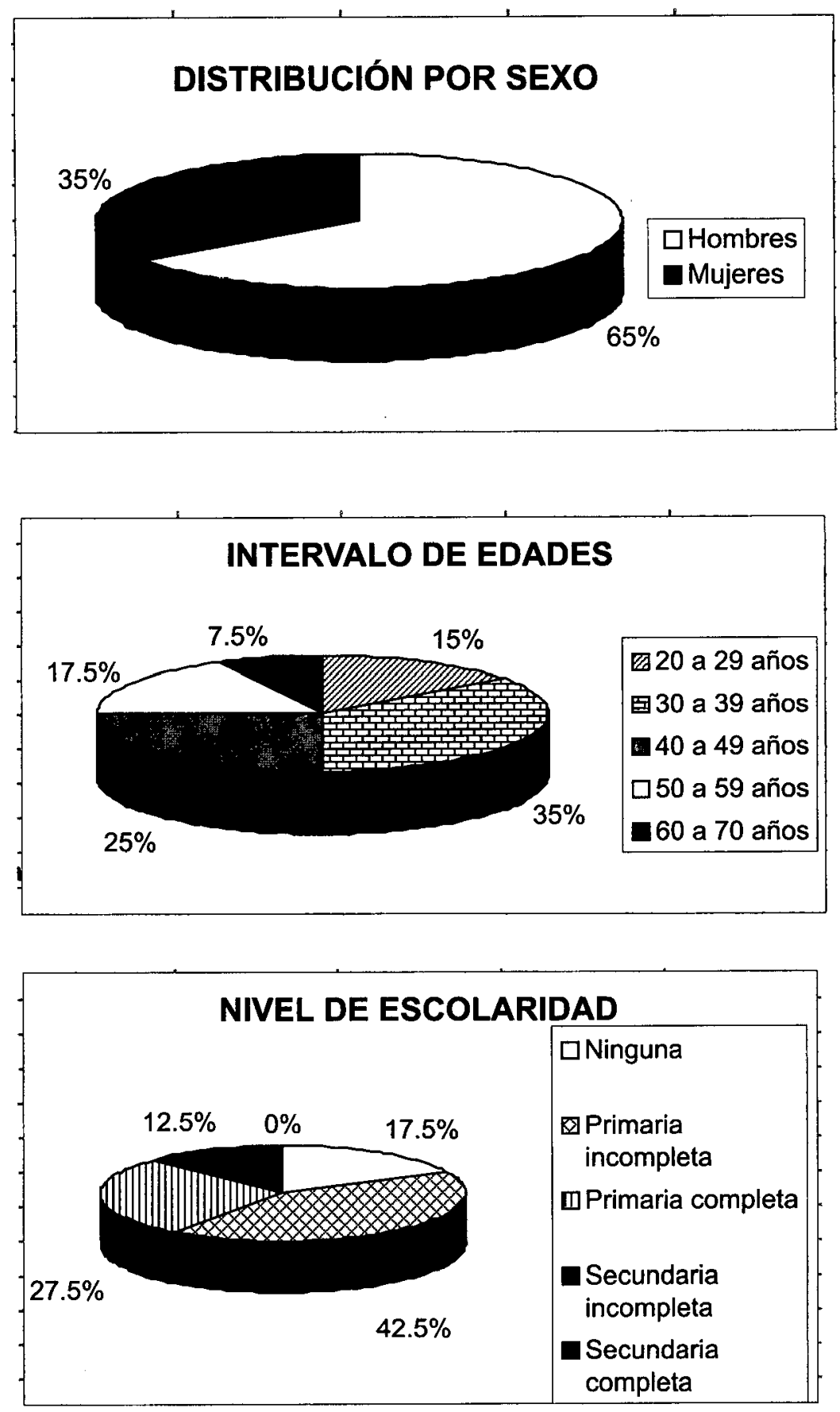

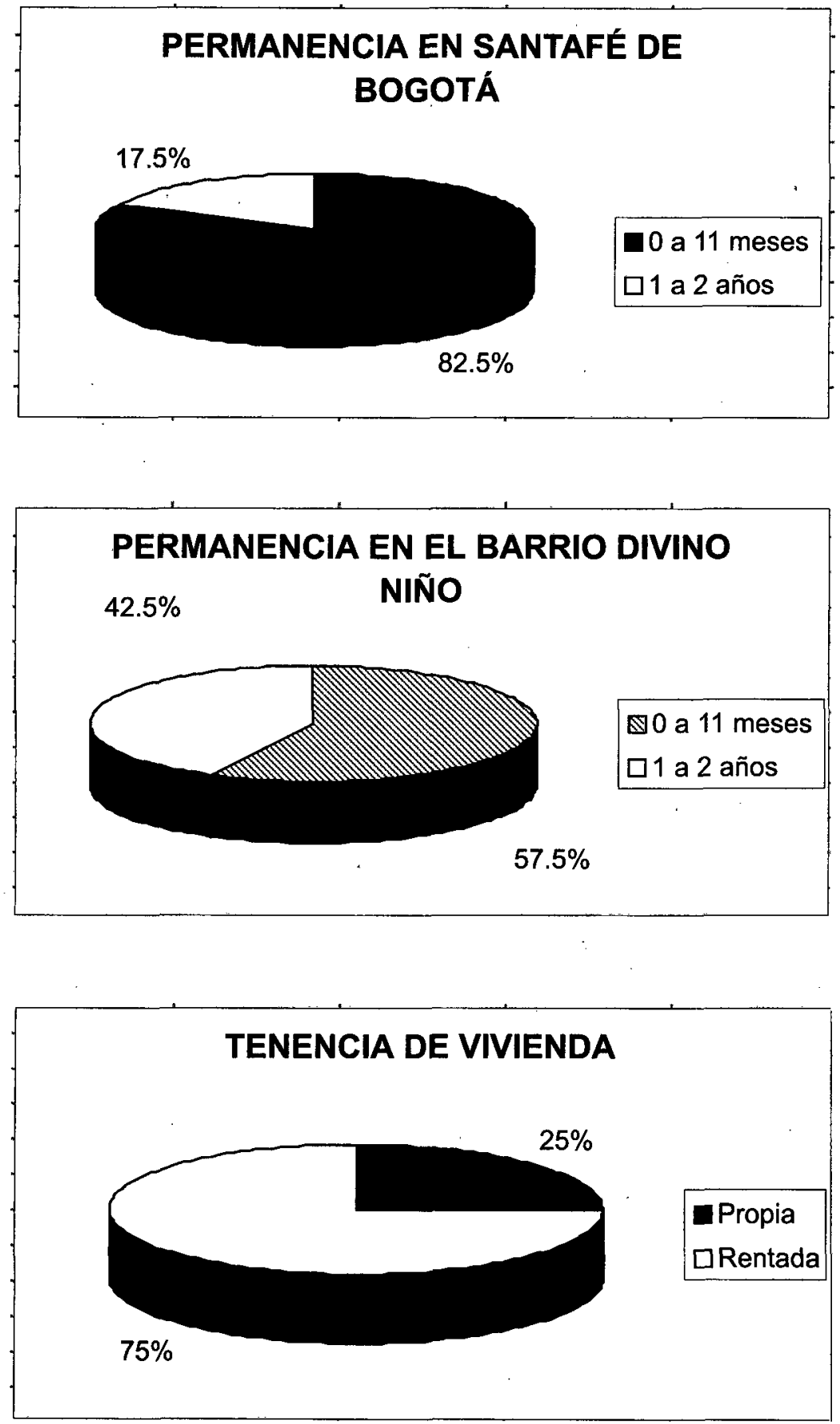

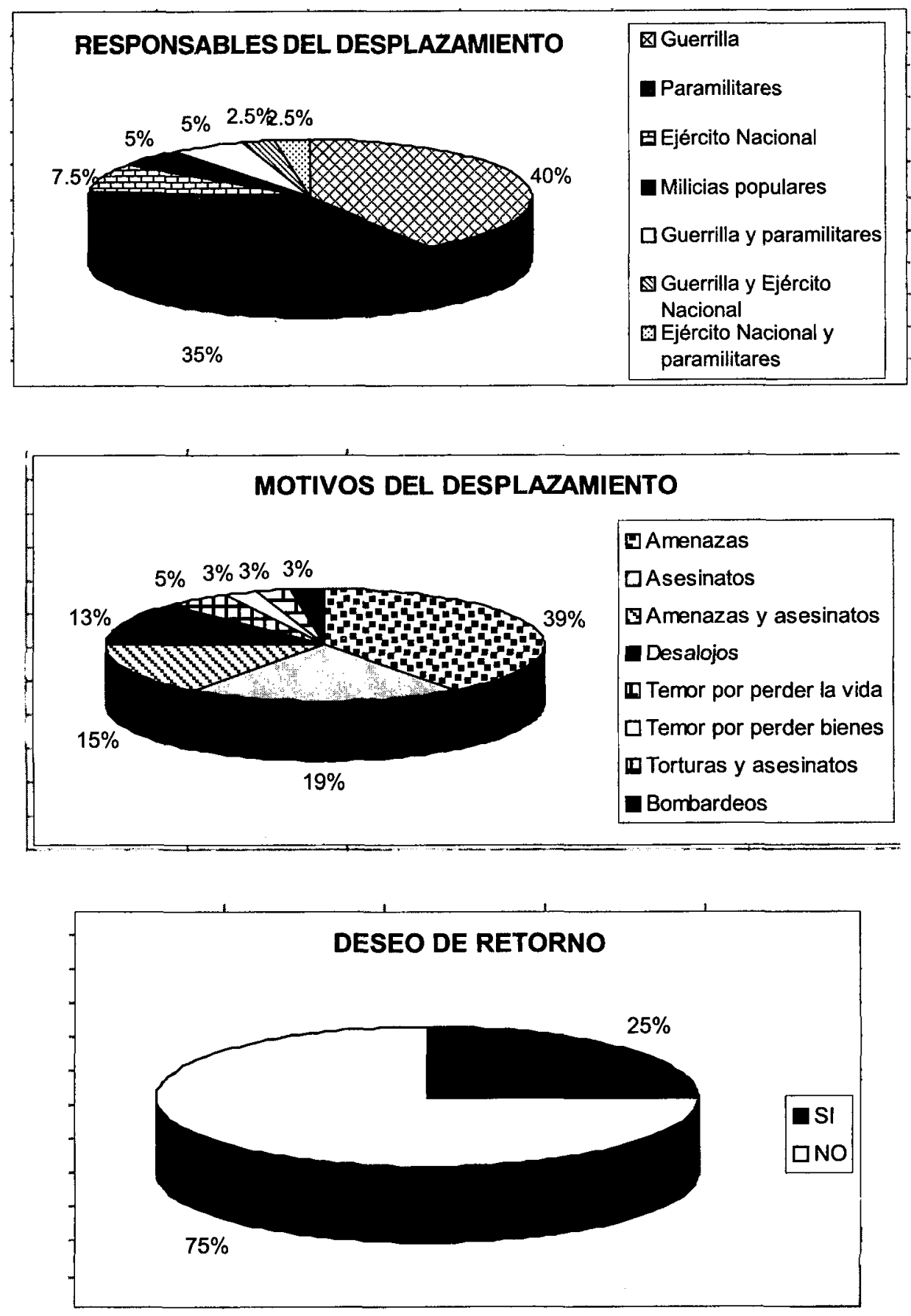


\section{CONCEPTUALIZACIÓN DE TERAPIA OCUPACIONAL}

La segunda caracterización de la población está basada en el análisis de los componentes del Modelo Teórico de la Ocupación Humana, planteado por Gary Kielhofner (2), que explica cómo está motivado, organizado y ejecutado el Comportamiento Ocupacional, definiendo éste como el conjunto de actividades en las cuales el individuo se involucra la mayor parte de su tiempo; estas actividades son de trabajo, juego y de la vida diaria, realizadas con base en sus creencias, preferencias, experiencias acumuladas, influencia del medio ambiente y patrones de comportamiento específico adquiridos. Este modelo conceptualiza el sistema humano en una jerarquía de tres subsistemas: Volición, Habituación y Ejecución; los tres representan una constante humana que va desde la ejecución automática de comportamientos diarios y rutinarios, hasta la elección consciente de roles ocupacionales.

Como consecuencia del desplazamiento forzado, las personas sufren cambios en sus estilos de vida en términos de cotidianidad, puesto que se ven obligadas a cambiar de un medio de vida generalmente rural a uno urbano con demandas culturales, sociales, económicas y laborales diferentes a las anteriores, lo que conlleva cambios abruptos en al ocupación dentro de los procesos de trabajo tiempo y tiempo libre, y en las expectativas de vida.

El proceso de readaptación a un nuevo estilo de vida posee un curso lento y sujeto a la aceptación consciente de las actuales circunstancias de vida, y trae consigo la dificultad de mantener un equilibrio en el Desempeño Ocupacional debido a que las prioridades, proyectos y metas cambian en su totalidad.

En el subsistema Volición, que hace referencia a los elementos que permiten una elección consciente para el Comportamiento Ocupacional, los cuales se traducen en la motivación necesaria para la realización de una conducta voluntaria, las personas desplazadas cambian sustancialmente sus motivaciones ocupacionales, suscribiendo éstas al reconocimiento del medio circundante, identificación de las demandas sociales y económicas del hábitat urbano, iniciando así la exploración de alternativas viables en términos de ubicación de vivienda y consecución de empleo para la supervivencia inmediata, para luego durante un proceso de readaptación satisfactoria y de mayor complejidad, dirigir sus acciones hacia el logro de atención de necesidades más amplias mediante la búsqueda de un trabajo que repre- 
sente mayores ingresos económicos, y que por tanto redunde en un mejoramiento de la calidad de vida posterior.

Dentro del subsistema Habituación, que abarca los roles y hábitos del ser humano, los cuales unen y guían la ejecución de patrones rutinarios de conducta, se encuentra el cambio de rol laboral no sólo en el jefe de familia, sino en los demás miembros; esto puede afirmarse al comprender el cambio que ocurre: Las familias provenientes en su mayoría de medios rurales, desempeñaban allí roles de trabajador, en el caso de los cabezas de familia, en oficios propios de la actividad económica sobresaliente del sitio de vivienda, entre los que se encuentran el cultivo de la tierra, el cuidado de ganado, la comercialización (venta) de producto del campo; oficios que al trasladarse a la ciudad ya no resultan rentables, puesto que no cuentan dentro de las actividades económicas de ésta; por tanto las demandas de empleo en estas áreas son nulas, lo que obliga al trabajador a buscar alternativas diferentes para su supervivencia y la de su familia, lo que enseguida denota un cambio de rol. Esta situación se agrava cuando el jefe de hogar es asesinado y/o cuando los menores desertan tempranamente de la escuela y se dedican a las actividades de tipo productivo.

En cuanto a los hábitos, se destaca la organización poco adecuada de las conductas rutinarias; esto se apoya en el hecho de que en un día típico el trabajo abarca la mayor parte del tiempo, aproximadamente doce horas, bien en trabajos varios o en uno sólo; se destina un tiempo adecuado para las actividades de autocuidado, pero en la estructuración del tiempo libre es observable un desequilibrio, ya que no son programadas a nivel individual o familiar actividades de esparcimiento variadas; el aprovechamiento del tiempo libre no se ve como un factor relevante.

Respecto al subsistema Ejecución, encargado de producir comportamientos con propósito a través del desarrollo de habilidades de diversos tipos, encaminadas a la obtención de un Comportamiento Ocupacional dinámico y funcional, que resuelve problemas y toma en cuenta las experiencias pasadas, se encontró que las víctimas del desplazamiento poseen habilidades poco efectivas para el Desempeño Ocupacional propio de la ciudad, puesto que éstas son aptas para el medio rural; por dicha razón, la población al asentarse en el medio urbano, no percibe metas futuras definidas para su vida y sus acciones son desorientadas, con presencia de un importante disbalance ocupacional. Las habilidades que poseen no son apropiadas para la resolución de los problemas que ofrece el nuevo medio por su complejidad; 
la desorientación inicial y el establecimiento de metas a corto y mediano plazo, no permiten una proyección hacia el futuro, sumándose a ello las precarias condiciones culturales, sociales, laborales y económicas en las que se desenvuelven.

La información acerca de los cambios en el Desempeño Ocupacional Laboral, uso del Tiempo Libre y expectativas de vida presentes en la población desplazada, se recoge en las siguientes tablas: (Ver anexos)

De la información condensada en las tablas, se concluye lo siguiente:

- Suceden cambios en el ámbito familiar, como efecto del desplazamiento forzado, observándose que la persona que generalmente asumía el rol de proveedor, pasa a ser uno más de los responsables de éste; incluso menores reemplazan o posponen sus estudios, para contribuir al sustento familiar.

- Se presentan cambios importantes en las ejecuciones ocupacionales de tipo laboral, puesto que tradicionalmente las personas desempeñaban labores propias del medio rural, experimentando satisfacción por su productividad; mientras que en el medio urbano las exigencias cambian, y por tanto las nuevas ejecuciones ocupacionales laborales se circunscriben a puestos de trabajo con demandas diferentes, y que por su naturaleza son de baja remuneración.

- El aprovechamiento del tiempo libre en el actual medio de vida es mayor, puesto que la ciudad ofrece variedad de opciones; mientras que en el medio anterior, la mayoría del tiempo es dedicado a las actividades de tipo laboral, y. las opciones de esparcimiento que ofrece éste, son limitadas.

- Se presentan bajas expectativas frente al nuevo medio de vida, puesto que la motivación mayor se dirige a la satisfacción de necesidades básicas; asimismo, se observa que las expectativas a largo plazo con respecto a mejorar la calidad de vida son pocas, debido a la inquietud frente al futuro, a lo que se suma una disminución en el sentido de pertenencia al contexto geográfico y al grupo social.

Es importante anotar que aunque la investigación fue realizada con una muestra poblacional determinada, los resultados en cuanto a cambios en la cotidianidad y estilos de vida, son generalizables a las personas desplazadas del territorio colom- 
biano, puesto que la dinámica del desplazamiento posee los mismos efectos para todas sus víctimas.

\section{REFLEXIÓN EN TORNO A LA INTERVENCIÓN EN POBLACIONES DESPLAZADAS}

El Terapeuta Ocupacional tradicionalmente se ha ubicado y ha sido reconocido socialmente dentro del enfoque de restauración y rehabilitación de la función, y ha intervenido en las áreas de promoción de salud y prevención de enfermedad, por lo que se hace necesario definir teórica y empíricamente el papel del profesional en nuevos campos de acción, "donde se requiere cada vez más su participación en los programas contra la violencia en todos los niveles, en aquellos destinados para las víctimas de la violencia colectiva, como campos de desplazados, hogares sustitutos, programas de bienestar, lugares de reclusión, indigentes y otros". (3)

En este sentido, la investigación es un inicio del abordaje por parte de Terapia Ocupacional en la temática de la violencia como problema social ponderante, donde se considera importante el desarrollo de habilidades y destrezas conceptuales y metodológicas que habiliten al profesional en la intervención de esta problemática, al aplicar los conceptos teóricos de modelos propios y generar conocimiento que explique la incidencia de la violencia y puntualmente del desplazamiento forzado, en el Comportamiento Ocupacional de las poblaciones víctimas de este flagelo, lo que representa un escenario propicio para la proyección profesional en esta área.

El Terapeuta Ocupacional actúa como un agente facilitador de procesos de crecimiento personal y social, potancializador y dinamizador de habilidades propendiendo por la readaptación social, y creador de las condiciones necesarias para analizar y solucionar los problemas relacionados con el Desempeño Ocupacional de grupos vulnerables como éste. (4)

Puesto que el objeto de estudio de Terapia Ocupacional es la Ocupación Humana, el profesional debe propiciar niveles de funcionalidad ocupacional a través de las variables de productividad, satisfacción personal y sentido de logro; asimismo, promueve la participación comunitaria con el fin de dar respuesta a las necesidades e intereses de la población donde interviene; en este proceso es de vital importancia el establecimiento de óptimas relaciones interpersonales con los miembros de la 
comunidad, asumiendo una actitud receptiva frente a sus necesidades y problemas, con el fin de contribuir de una manera eficaz a la resolución de los conflictos que se presentan en este ámbito social. (5).

En referencia a las disposiciones legales que contemplan la atención integral a personas desplazadas por la violencia, existe una importante contribución de las Organizaciones No Gubernamentales y Entidades de carácter nacional e internacional, que prestan servicios asistenciales a corto y largo plazo, promocionan la organización comunitaria, y defienden y protegen los Derechos Humanos. Entre estas se destacan los programas adelantados por la Iglesia Católica, la Conferencia Episcopal Colombiana, el Centro de Investigación y Educación Popular (CINEP); a nivel internacional, la Cruz Roja, el Alto Comisionado de las Naciones Unidas para los Refugiados (ACNUR), la Organización Panamericana de la Salud (OPS) y la Organización de Estados Americanos (OEA), poseen una amplia trayectoria en el manejo y tratamiento de la temática en cuestión. (6) (7). Es de lamentar que en Colombia aún no se de cabal observancia a las normas dictadas por la comunidad internacional, para regular la conducción de la hostilidad y proteger a las víctimas de los conflictos armados.

Las políticas estatales basadas en la Ley 387 de 1997, que pretende garantizar la prevención, atención, protección, consolidación y estabilización socioeconómica de los desplazados internos en Colombia; asimismo, enmarca las acciones planteadas dentro del Sistema, Programa y Plan Nacional de Atención Integral a la Población Desplazada por la Violencia. (8)

Las acciones gubernamentales involucran a entidades estatales que por su naturaleza jurídica, están en la capacidad de ofrecer ayuda en áreas específicas, como salud, educación, empleo, vivienda, alimentación, entre otros; para tales fines, estamentos como el Ministerio del Interior, Agricultura y Desarrollo, SENA, INURBE, INCORA, Caja Agraria, principalmente, poseen recursos humanos y financieros destinados a formular e implementar acciones en materia. (6)

Estas determinaciones legales han demostrado un pobre desarrollo de verdaderas políticas encaminadas a solucionar con eficiencia y eficacia los efectos del desplazamiento, puesto que la oferta ha alcanzado una mínima parte de la dimensión contemplada en su normatividad, los planes de consolidación y estabilización socioeconómica poseen resultados muy limitados, y en numerosas ocasiones di- 
chas determinaciones han entorpecido el trabajo de importantes Organizaciones No Gubernamentales tanto locales como extranjeras. A esto se suma las continuas violaciones de derechos humanos por parte de los servidores públicos, que al abusar de su autoridad violentan indiscriminadamente a la población civil.

\section{RECOMENDACIONES}

- Planear estrategias de acción para la población desplazada, a través del diseño, oferta e implementación de programas de Terapia Ocupacional dirigidos a promover un balance ocupacional y a la adquisición de estilos de vida saludables, dentro de un marco de trabajo institucional.

- Involucrar la educación superior en la temática del desplazamiento en términos formativos, con el fin de sensibilizar al recurso humano y de esta forma aportar desde la perspectiva profesional, soluciones de utilidad práctica a tal fenómeno.

- Dar continuidad a la investigación científica en temáticas sociales acordes con la realidad del país, con el fin de optimizar la atención a grupos vulnerables, concatenando prácticas profesionales con grupos interdisciplinarios para abordajes con mayor integralidad.

- Capacitar a la población en cuanto a la apropiación de la ley existente en materia, y para el trabajo mediante la creación de talleres protegidos y convenios con entidades de capacitación regionales, con el fin de proporcionar a esta población elementos para optimizar su reinserción sociolaboral, buscando una progresiva vinculación a la vida productiva.

- Optimizar la oferta estatal, en la búsqueda de programas y políticas con mayor eficacia y efectividad, para la atención de las poblaciones desplazadas por violencia, donde los estamentos gubernamentales involucrados apoyen dicha causa, mediante un mayor compromiso institucional. 


\section{BIBLIOGRAFÍA}

1. CÁRDENAS, Maricela. "Desplazados". La República. Santa Fe de Bogotá: Julio 5 de 1998.

2. ASOCIACIÓN COLOMBIANA DE TERAPIA OCUPACIONAL. Contenido descriptivo del Modelo Ocupación Humana de Gary Kielhofner. Santa Fe de Bogotá: Noviembre de' 1995.

3. CAICEDO CONCHA, María Eugenia. "El Terapeuta Ocupacional y la rehabilitación en salud mental". En: Revista Ocupación Humana / Asociación Colombiana de Terapia Ocupacional. Vol. 7, №. 1. Santafé de Bogotá: 1997.

4. RODRÍGUEZ, María Teresa. Modelo de actuación del Terapeuta Ocupacional en comunidad. Universidad Nacional de Colombia. Santafé de Bogotá: 1992.

5. RODRÍGUEZ, María Teresa. "Competencias ocupacionales y desarrollo comunitario: Un modelo operativo". En: Revista Ocupación Humana / Asociación Colombiana de Terapia Ocupacional. Vol. 3, №. 1. Santafé de Bogotá: 1989.

6. DELEGACIÓN DE LA COMISIÓN EUROPEA PARA COLOMBIA Y ECUADOR (ECHO). Todos tenemos derecho a una vida digna: Oferta de atención a la población desplazada. Santafé de Bogotá: 1998.

7. HERNÁNDEZ, Esperanza, et al. Identificación de la Oferta para la Atención a la Población Desplazada por la Violencia Política en Colombia. Santa Fe de Bogotá: 1998.

8. PRESIDENCIA DE LA REPÚBLICA. Ley 387 del 18 de Julio de 1997. Santa Fe de Bogotá: 1997. 


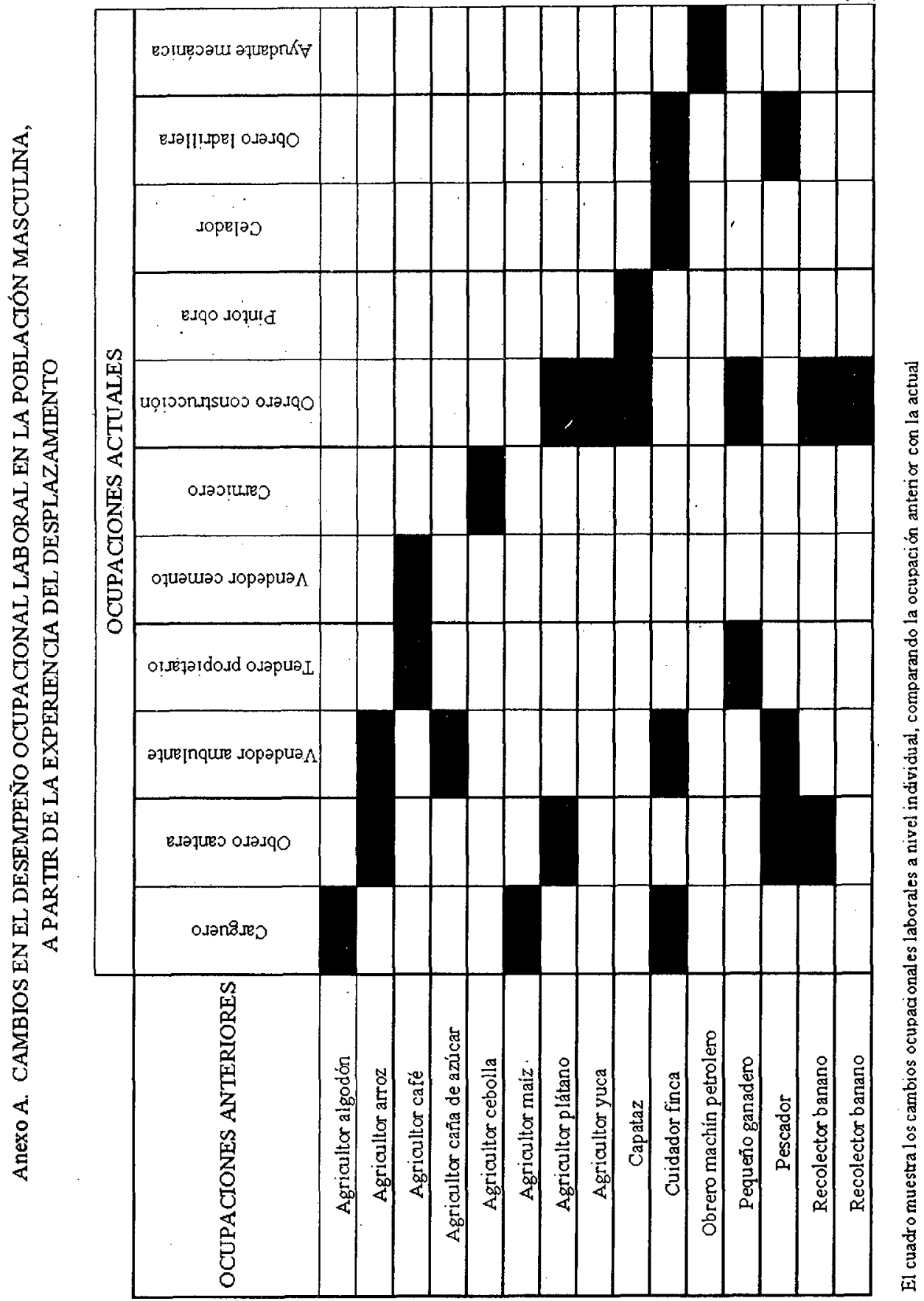




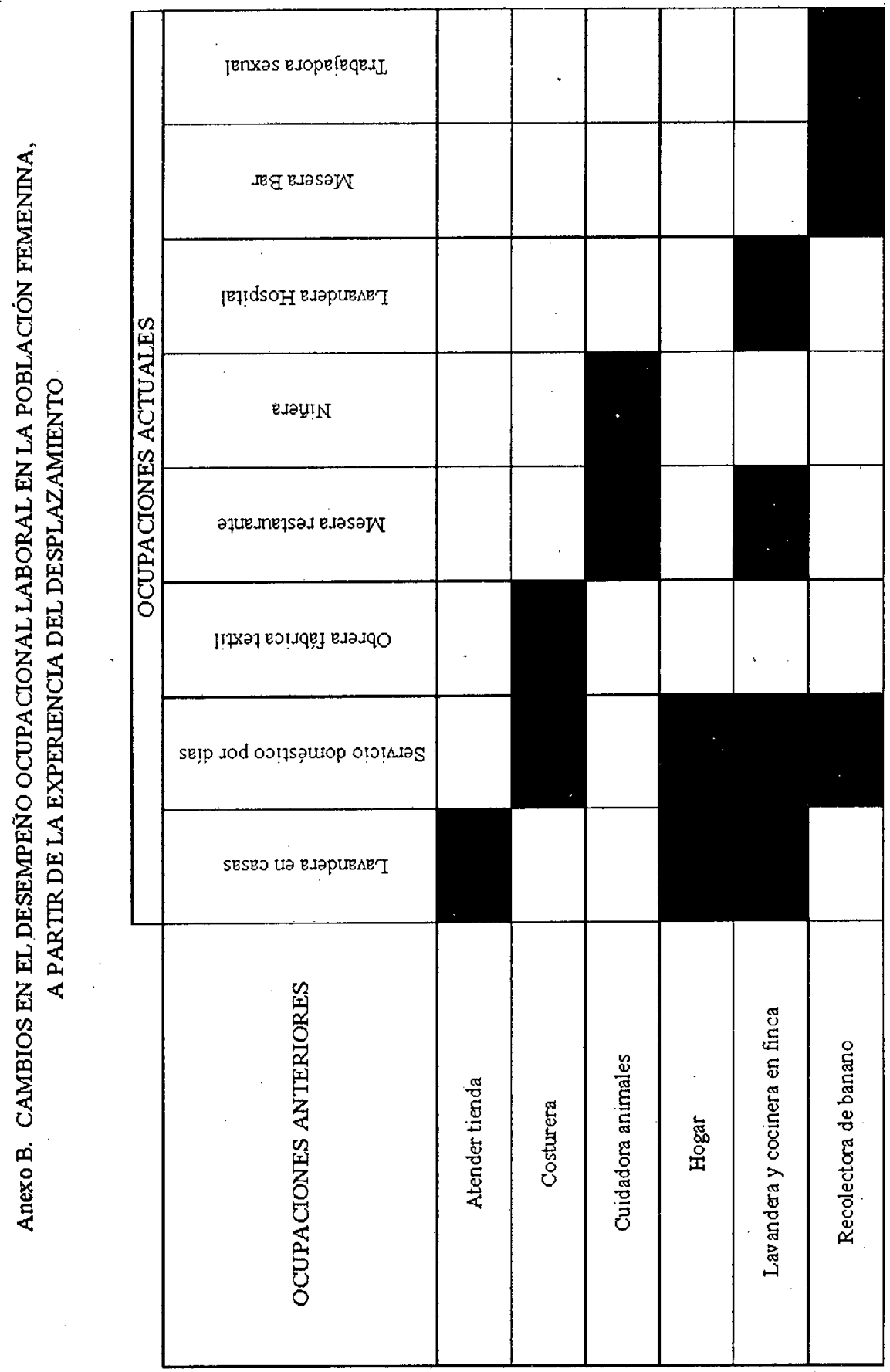




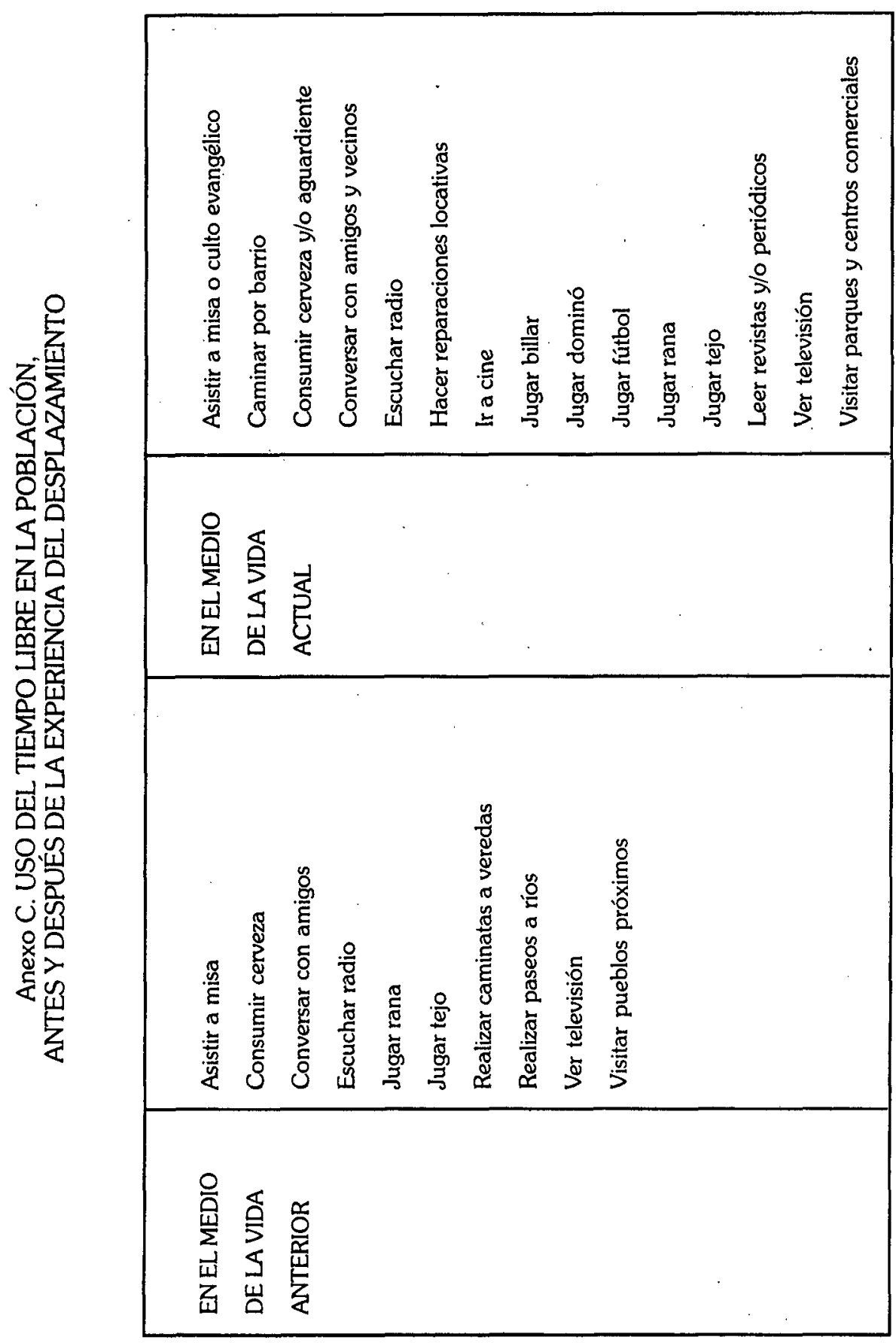




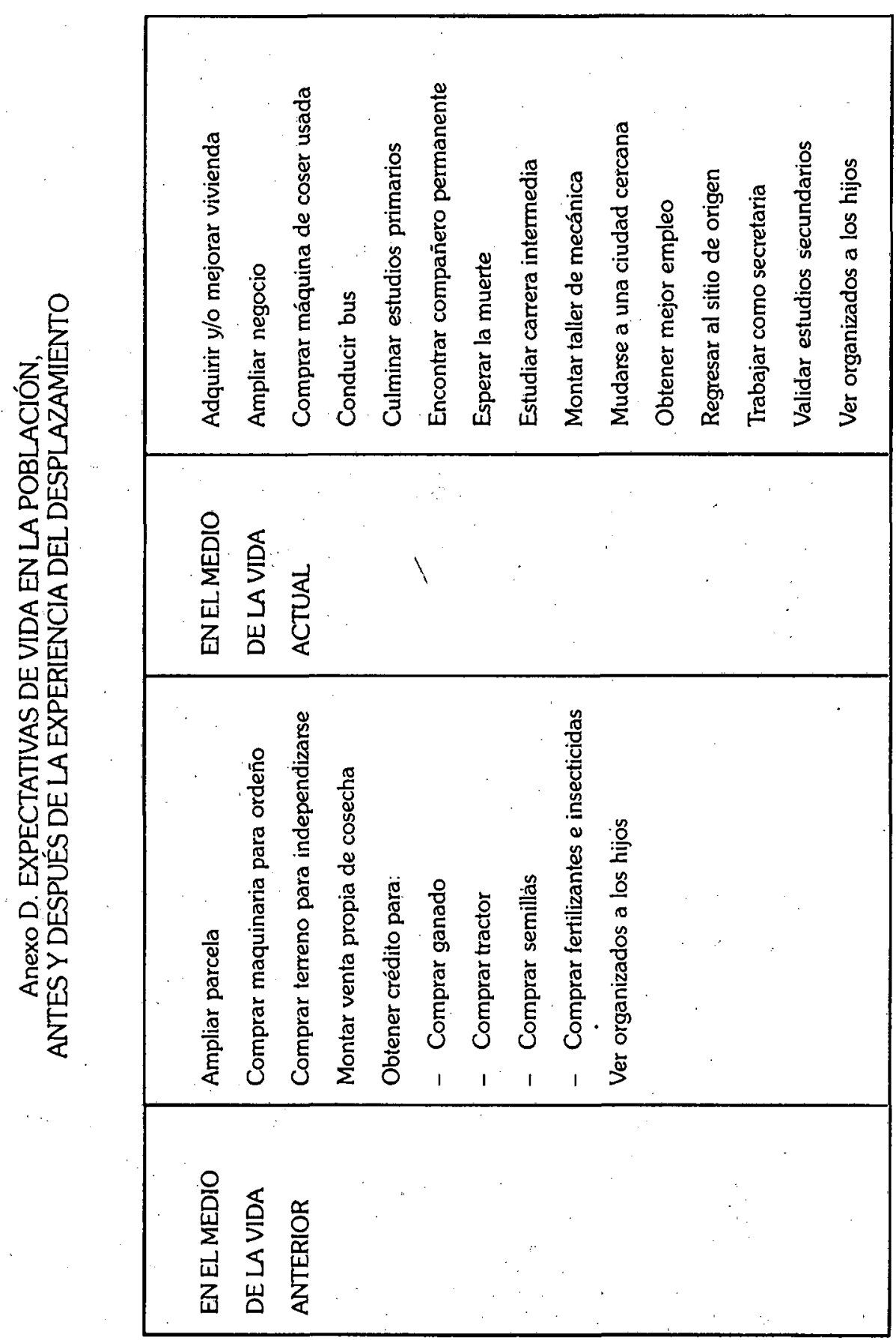

